\title{
THE IMPACT OF CLEANING WEIGHTS BEFORE CALIBRATION
}

\author{
Christian Müller-Schöll ${ }^{1}$ \\ ${ }^{1}$ Mettler-Toledo International Inc., Greifensee, Switzerland, christian.mueller-schoell@mt.com
}

\begin{abstract}
:
This paper investigates the necessity of cleaning weights before calibration. The conflict between cleaning a weight and breaking the history by cleaning is analyzed. Experience from many hundreds of calibrations with measurements before and after cleaning is reported and analyzed. It is concluded, that weights should be cleaned in order to reach a stable state but should also be calibrated before and after cleaning to gain information about metrological traceability and thus the validity of the results that were produced with the weights.
\end{abstract}

Keywords: calibration; weights; cleaning; contamination; OIML R111-1; traceability

\section{INTRODUCTION}

Cleaning of physical material measures for mass has been a topic since the making of the first kilogram standards in the $18^{\text {th }}$ century.

This paper details on the necessity and the effects of cleaning of industrial weights which are used for testing or calibration of weighing instruments. For this, a pool of some hundred calibrations of weights before and after cleaning are investigated.

Section 2 sets the background for the historically documented need for cleaning weights. Section 3 treats the question if cleaning requires calibrations before and after, while section 4 raises the question of safety that is gained by cleaning. Section 5 gives a practical example of a laboratory's policy regarding cleaning of weights. In section 6 we detail the results of one specific weight, while in section 7 we shed light from different angles on the question of significance. In section 8 we present results of some hundred weights' calibrations and in section 9 we investigate the effect of cleaning on metrological traceability. Section 10 gives an overview of the conclusions.

\section{BACKGROUND FOR CLEANING WEIGHTS}

From the beginning of modern mass metrology in the $18^{\text {th }}$ century, metrologists have considered cleaning of mass artefacts: even the IPK, which served as the definition of the kilogram until 2019, assumed its exact mass only after a cleaning and washing procedure (French: "nettoyage-lavage") which apparently removed as much as $15 \mu \mathrm{g}$ from the artefacts at the BIPM [1].

Obviously, the artefacts reach a stable mass value only in a "clean" state whereas the "unclean" state is instable, unreliable and dependent on external influences like time since last cleaning, intensity, frequency, and kind of usage etc.

Still today, in OIML R111-1, the step "cleaning weights" is listed as a mandatory element in the procedure for the assessment of conformity and OIML states "It is important to clean weights before any measurements are made..." [2]. However, no specific method is prescribed. The calibration or verification laboratory may choose appropriate methods according to their expertise, competence and capabilities.

Although the definition of the kilogram has been freed from being dependent on an artefact in 2018, weight pieces are still an indispensable device for establishing a mass scale, for calibrating weights and for calibrating and testing weighing instruments.

It is worth noting that immediately after cleaning the surfaces of weights are likely to be in an unstable state. Therefore, it is necessary to allow for a certain stabilisation period (B.4.2 in [2]) whose length is dependent on the cleaning method and the accuracy class of the weights.

The reason for cleaning is obviously the fact that weight pieces are frequently contaminated and that the "clean state" is the only reliable and reproducible state, while the "dirty state" is rather volatile. In fact, the mass of the dirty state can be reduced as unintentionally (by randomly wiping away dirt) as it can be raised (by touching or contaminating the weight). With a reliable and reproducible and non-abrasive cleaning method, it should be possible to bring a piece of stainless steel always to the same mass (within measurable reproducibility).

\section{IS CLEANING AN INTERVENTION?}

ISO 17025 requires that calibration results shall be collected, "before and after any adjustment or repair, if available" [3]. The intention of this 
requirement is obviously that an uninterrupted history of the device under calibration is available and the user of the object can use the information of the "as found" state in order to assess the appropriateness of handling, of assumed accuracy and stability and finally of calibration intervals. Unfortunately, [3] limits the applicable cases in an exhaustive list to "adjustment" and "repair". It is clear that a cleaning intervention on a weight might have the same effect as an adjustment intervention and thus should be considered in the same way.

The strict application of the ideas of [3] together with the procedure given in [2] must lead to the conclusion that all weights have to be cleaned before calibration and all calibrations have to consist of a set of two full calibrations, one before and one after the cleaning. The according calibration certificate is then a so-called "as-foundas-left-certificate". Not collecting data of the state before cleaning would - strictly speaking - result in a "break in the history" of the measuring instrument.

\section{SAFETY CONSIDERATIONS}

The fact that measurement instruments can carry over any kind of contamination from the environment they are used in, into the calibration laboratory, is frequently under-estimated, according to the view of the author. This applies for both hazardous contaminations as well as for "just dust and dirt" in the case of weight pieces. Both kinds of contaminations mentioned above endanger the integrity of the calibration laboratory equipment, and, even more important, endanger the integrity of un-involved third parties' equipment, when contamination is carried from an instrument onto the laboratory equipment and from there to third party customers' instruments.

\section{THE POLICY OF THE SWISS CALIBRATION LAB OF METTLER TOLEDO}

The Laboratory of Mettler-Toledo GmbH in Switzerland calibrates many thousands of weights per year. Customers expect calibration values of a stable state of their weights. Unfortunately, most customers are not willing to pay for the effort of a double calibration (before and after cleaning).

As a compromise between the pure metrology of not changing instruments without additional calibration and the minimisation of the risk of contamination, and the request of low prices and stable and consistent calibration results, the policy generally applied for weight calibrations is as follows (and this policy is made known to the customer):

- Weights will be cleaned generally to protect the laboratory and un-involved parties' artefacts.
- $\mathrm{E}_{1}$ class weights will not be cleaned.

- If a customer wants a calibration of the uncleaned state, the risk for the laboratory is estimated. In severe cases, the bottom surface of the weight is cleaned in order to protect the comparator balances from dirt. The customer may choose if the weight shall be cleaned and calibrated again.

\section{EFFECT OF CLEANING WEIGHTS}

In contrast to most customers of the calibration laboratory, there is one big company which, as a general policy, requests the cleaning of weights together with a double calibration, before and after the cleaning. This company is in the pharmaceutical industry and is therefore strictly regulated. The management is used to issuing internal procedures and their staff is used to strictly following such procedures.

We have monitored some of these calibrations over a period of approximately ten years.

Although the SOP strictly advises the personnel not to touch the weights and use clean tools to handle the weights, these weights show clear signs of contamination.

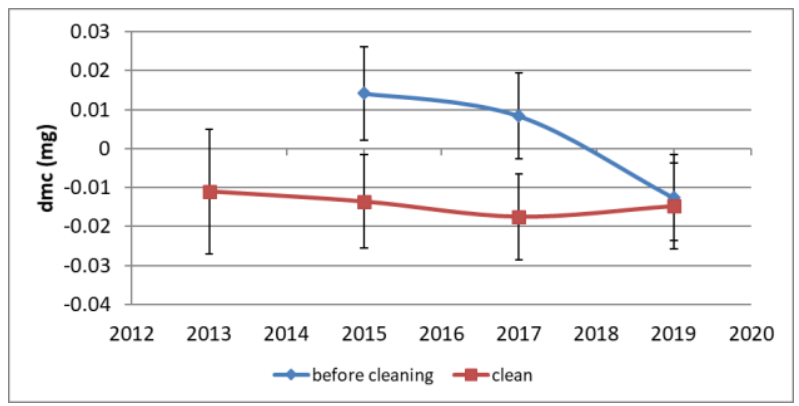

Figure 1: Typical calibration history $\left(50 \mathrm{~g}, \mathrm{E}_{2}\right)$ blue (upper) line shows deviation from nominal before cleaning; red (lower) line are values after cleaning. Uncertainty bars $95 \%$.

Figure 1 shows as an example a typical history of such a weight: the weight was first produced and calibrated in 2013 in a "clean" state. It was then recalibrated every two years with a calibration before (blue) and after (red) cleaning. In 2015 and 2017, the effect of cleaning is clearly visible: the values before cleaning are significantly higher. After cleaning, the values always come to the same stable level (within uncertainties).

For the calibration of 2019, no contamination was detected: either the handling of the weight has changed significantly or the weight has not been used (this information was not available).

The cleaning was done in a fully automated procedure with a heated bath with ultrasound cleaning using a regular, neutral industrial cleaner for stainless steel while constantly moving the weights. After cleaning, the weights are rinsed 
multiple times in a cascade style process in filtered and de-ionised water and let stabilise and acclimatise according to [2]. The difference before and after cleaning of the same year is about $-25 \mu \mathrm{g}^{1}$ for the $50 \mathrm{~g}$ weight, but varies between recalibrations. This means that the cleaning procedure has removed contamination of as much as $25 \mu \mathrm{g}$ mass from the weight of $50 \mathrm{~g}$ nominal mass.

There are two proofs that the cleaning did not remove any metal material from the weight (as one could suspect) but did effectively remove only dirt: first, all the "clean" values are consistent within their uncertainties. Second, we use the same type and material of weights in our calibration laboratory on robotic comparators as reference standard weights. For these weights, we do not observe any significant change in mass, neither over time nor before and after cleaning.

We conclude, the weight pieces (would they be left un-touched) are stable with time and our cleaning procedure does not affect the pure metal mass of the weight pieces in any detectable way.

\section{IS THE CONTAMINATION SIGNIFICANT?}

Depending on the point of view, there are multiple answers to the question if such a contamination is significant.

\subsection{Significance considering Calibrated Values}

Referring to Figure 1, it is obvious that, since the uncertainty bars $(95 \%)$ of approximately $12 \mu \mathrm{g}$ do not overlap, the difference in the values of 2015 and 2017 are significant. En-values amount up to -1.6 which, from the metrological view of the calibration laboratory clearly indicates that the values before and after cleaning are inconsistent and the amount of dirt removed therefore is significant.

\subsection{Significance if used "per Accuracy Class"}

One could also consider how the weights are used in the customer's process: these weights are used for routine testing of balances. The requirements for the weights coming from the customer's procedure is the adherence to class $\mathrm{E}_{2}$ according to [2]. If the En-value is calculated using the MPE value of $100 \mu \mathrm{g}$ instead of calibration uncertainty, En amounts to no larger than -0.2. Thus, it could be concluded that for the process of the application, the mass of contamination is insignificant since the weight value is allowed to vary within the class limits during its service life without affecting the balance test result.

\footnotetext{
${ }^{1}$ For the mass of removed contaminants as well as for correspondingly calculated En-values, we maintain negative values in this publication.
}

\subsection{Significance for Quality Control Interests}

The results are a clear sign that the instructions in the SOP ("handle weights with clean tweezers or with clean gloves") are not effective or not effectively executed. Thus it can be concluded, that the mass of the contamination is significant.

\subsection{Significance if Trends are considered}

Should the balance testing results be analysed not only for passing or failing a limit test, but also for trends over time, then the contamination of the test weight might superpose the actual behaviour of the balance. Should trends in the order of size of the contamination be relevant, then the contamination might be significant. (The one example given in section 6 might be considered irrelevant if the weights are used e.g. on a balance with a readability of $10 \mu \mathrm{g}$ and the amount of contamination is only a small multiple of the readability.)

The considerations above underpin the general rule that only the user of the measurement device can make the final decision on the relevance of the data for his specific application.

\section{GENERAL VIEW}

\subsection{En-Values of a Pool of Calibrations}

In our laboratory, we have observed some hundred calibrations of a pharmaceutical company with values before and after cleaning. The general picture is as follows:

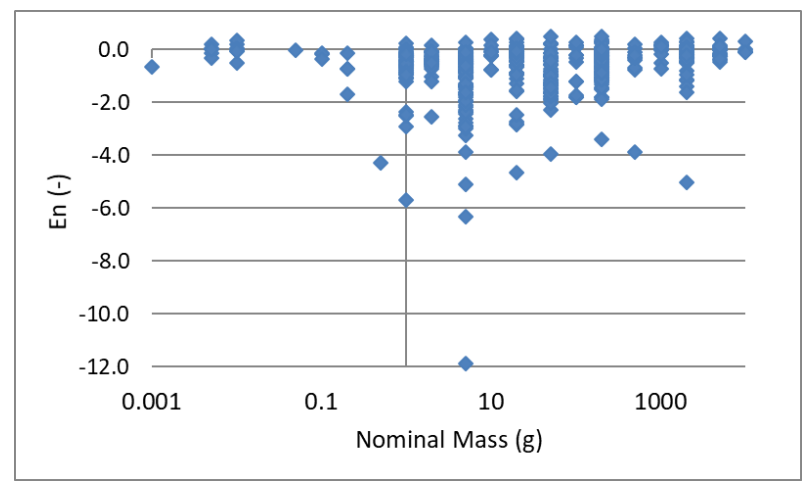

Figure 2: $E_{\mathrm{n}}$-values vs. nominal mass

As can be seen from Figure 2, we found many Envalues in the region between -1 and -2 . As explained above, this is metrologically significant, but probably insignificant for the actual application. Nevertheless, there is a wide variation and there were some results where the cleaning resulted in Envalues greater than -3 and one even larger than -10 (this was a $5 \mathrm{~g}$ weight). It is unknown unfortunately, how the customer reacted to these results.

There are also some positive En-values (the weight values appeared heavier after cleaning). 
These are owed to the reproducibility of the procedures of the calibration laboratory when obviously no significant contamination was present.

\subsection{Absolute Mass of Contaminants}

Another view on the pool of values is the absolute amount of contamination. Figure 3 shows the washed off contaminants' mass vs. the nominal mass of the pieces.

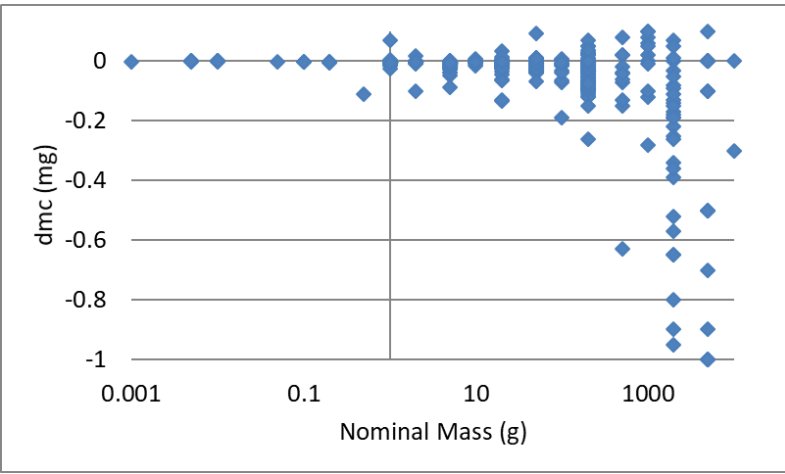

Figure 3: Absolute mass of contaminants vs. nominal mass

It is obvious that with increasing mass (or probably rather with increasing surface), the mass of contaminants rises: a $1 \mathrm{mg}$ piece shows hardly any contamination, while large contaminations occur for the largest weights. Furthermore, the more difficult to handle, the more contaminations occur. This holds especially true for weights of $2000 \mathrm{~g}$ and above.

According to the impression of the author, this is owed to improper manual handling of the weights with improper (or even without) tools or inappropriate gloves. It is certainly not a metallurgical or corrosive surface effect since other weights of the same type under our own control behave absolutely stably (see section 6).

\section{POSSIBLE LOSS OF TRACEABILITY}

During a calibration, metrological traceability to the reference is established (definition 2.41 in [4]).

During the service life of the calibration data (i.e. the calibration interval), traceability has to be maintained. This can be done by a combination of and not limited to: (1) intermediate checks using a check standard, (2) adding a component of uncertainty that accounts for assumed changes (e.g. $u_{\text {inst }}$ in [2]) or (3) a predictive modelling of the behaviour of the instrument.

At the end of the calibration interval, a calibration must be performed in order to confirm that traceability has persisted throughout the interval. A possible means of doing this is an Entest where the value and uncertainty assumed during service life are compared to value and uncertainty of the confirming end-of-interval calibration. If the confirmation of traceability fails, an according investigation is mandatory (e.g. "nonconforming work" in [3]). Possible consequences could be reconsidering the uncertainty assumptions or reconsidering the length of the calibration interval.

It is obvious that for the example of weight pieces, the confirming calibration at the end of the interval must be done in a not-cleaned state, exactly in the state the weights were used until their last day in service. Cleaning of the weight piece and the following calibration are then used to again establish traceability for the interval to come.

\section{CONCLUSIONS}

From these findings, we draw the following conclusions:

- The topic of cleaning of weights has been introduced historically, but is still legitimate today.

- From an analysis of many weights from a highly regulated environment and used by trained personnel, we showed that contaminations occur frequently, even when there are clear instructions (SOPs) which should prevent contaminations.

- Contaminations (in absolute units) tend to be large for weights of larger size.

- Only the user of the weights - with deep metrological knowledge of the process in which the weights are being used - can answer the question if the amount of contamination is significant or relevant.

- OIML R111-1 neglects the topic of calibrating before and after cleaning.

- ISO 17025 is unclear (or incomplete) in listing cases in which a "before and after" calibration is mandatory and thus leaves the responsibility of the decision to the customer.

- Metrological traceability is endangered by improper handling. Measures must be taken to maintain traceability during the calibration interval.

- Calibrations before and after cleaning ("as found" and "as left") are inevitable to gain all information necessary for appropriate management of weights as a metrologically traceable measuring device and to confirm traceability of the past interval.

\section{SUMMARY}

In this paper, we have presented the importance of cleaning weight pieces. Calibrations should be performed before and after cleaning in order to obtain information about the behaviour of the devices, about the effects the usage has, about the appropriateness of the uncertainty and of the calibration interval. The information from both calibrations is indispensable for establishing, 
maintaining and confirming metrological traceability.

\section{REFERENCES}

[1] M. Stock et al., "Calibration campaign against the international prototype of the kilogram in anticipation of the redefinition of the kilogram part I: comparison of the international prototype with its official copies", Metrologia, vol. 52, pp. 310-316, 2015.

[2] OIML R111-1, Organisation Internationale de Métrologie Légale, Paris, France, 2004.

[3] ISO/IEC 17025, International Organization for Standardization, Geneva, Switzerland, 2017.

[4] JCGM 200, International vocabulary of metrology - Basic and general concepts and associated terms (VIM), BIPM et al., 2012. 